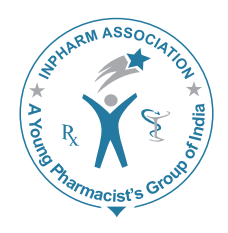

\title{
JVP
}

\section{Oxidative Stress Induced by Fluoroquinolones on Treatment for Complicated Urinary Tract Infections in Indian Patients}

\author{
Talla V, Veerareddy PR ${ }^{1}$ \\ Department of Pharmacology and Toxicology, National Institute of Pharmaceutical Education and Research \\ (NIPER), Hyderabad, 'Department of Pharmaceutics, St Peters' Institute of Pharmaceutical Sciences, \\ Warangal, Andhra Pradesh, India
}

Address for correspondence: Prof. Prabhakar Reddy V; Email: vpreddyindia9@gmail.com

\begin{abstract}
The aim of the study is to examine the oxidative stress in patients on fluoroquinolones (ciprofloxacin, levofloxacin, gatifloxacin) therapy for complicated urinary tract infections and to correlate with plasma concentrations at different time intervals. Superoxide dismutase (SOD), glutathione, plasma antioxidant status and lipid peroxides were evaluated in 52 patients on different dosage regimens up to 5 days. There is significant and gradual elevation of lipid peroxide levels in patients on ciprofloxacin $(3.6 \pm 0.34 \mathrm{nmol} / \mathrm{ml}$ to $6.2 \pm 0.94 \mathrm{nmol} / \mathrm{ml})$ and levofloxacin $(3.5 \pm 0.84 \mathrm{nmol} / \mathrm{ml}$ to $5.1 \pm 0.28 \mathrm{nmol} / \mathrm{ml})$ dosage regimen but not with gatifloxacin $(3.5 \pm 0.84 \mathrm{nmol} / \mathrm{ml}$ to $3.74 \pm 0.17 \mathrm{nmol} . \mathrm{ml})$. There was substantial depletion in both SOD and glutathione levels particularly with ciprofloxacin. On the $5^{\text {th }}$ day of treatment, plasma antioxidant status decreased by $77.6 \% \%, 50.5 \%, 7.56 \%$ for ciprofloxacin, levofloxacin and gatifloxacin respectively. In conclusion ciprofloxacin and levofloxacin induce more reactive oxygen species that lead to cell damage than gatifloxacin irrespective of their concentrations in patient population.
\end{abstract}

Key words: Dosage regimen, 2,2-diphenyl-1-picrylhydrazyl, fluoroquinolones, lipid peroxides, superoxidedismutase

\section{INTRODUCTION}

Fluoroquinolones are most widely used today for the treatment of bacterial infections belongs to urinary tract or respiratory tract and are considered safe and well-tolerated antibacterial drugs. The name fluoroquinolone comes

\begin{tabular}{|l|l|}
\hline \multicolumn{2}{|c|}{ Access this article online } \\
\hline Quick Response Code: & \multirow{2}{*}{ Website: } \\
\hline & www.jyoungpharm.in \\
& \\
\hline
\end{tabular}

from the presence of fluorine at $\mathrm{C}-6$, which is in the left-sided ring. Fluorine is found in all the modern agents. A cyclic diamine R2 usually is present at the 7-position. Some variation is permitted at C-5 and C-8. Newer agents have had substitutions of amino or methyl at the C-5 site. At the 8-position, numerous small substituents, such as fluorine, chlorine and methoxy, has been found to improve potency. In general, these antimicrobial agents have high bacteriological and clinical cure rates among most uropathogens and low rates of resistance among common uropathogens. ${ }^{[1]}$

Ciprofloxacin (CFX) is a second generation fluoroquinolone antibiotic introduced to clinical therapy in 1987. 
Ciprofloxacin resulted from the replacement of N-1 ethyl group of norfloxacin by cyclopropyl group which resulted in extensive anti-bacterial spectrum ${ }^{[2-5]}$ and the reason for being used widely in variety of human infections. ${ }^{[6]}$

Levofloxacin (LVX) is a third generation fluoroquinolone antibiotic and is the optical S-(-) isomer of the racemic drug substance ofloxacin. It has a broad spectrum of in vitro activity against Gram-positive and Gram-negative bacteria, as well as certain other pathogens such as Mycoplasma, Cblamydia, Legionella and Mycobacteria spp. Levofloxacin is significantly more active against bacterial pathogens than $\mathrm{R}-(+)$-ofloxacin. ${ }^{[7]}$

Gatifloxacin (GTX) is active against Gram-positive and Gram-negative organisms, including anaerobes such as, Mycoplasma, Chlamydia, and Legionella and mycobacteria. ${ }^{[8-10]}$ Like other quinolones, gatifloxacin penetrates well into leukocytes, which can deliver active drug to sites of infection and play an important role in the treatment of intracellular pathogens.

Nausea, diarrhea, vomiting and similar symptoms were the most common side effects noted during therapy with fluoroquinolones $(0.8 \%$ to $6.8 \%)$. Central nervous system-related adverse drug reactions vary from mild (headache, dizziness, tiredness or sleepiness) to severe (psychotic reactions, hallucinations, depression, and seizures). The elder patients are susceptible to the central nervous system effects of these agents, with dizziness being a particular concern. All quinolones have some photosensitivity potential including Ciprofloxacin especially in higher doses.

During clinical trials, the overall frequencies of adverse effects associated with to vary between 4.4 and $20 \% \cdot{ }^{[11,12]}$ One of the most important concerns is its tendinitis potential and rupture of the Achilles tendons. The tendon rupture can occur with short-term use and small dose. The pathophysiology of this adverse drug reaction by fluoroquinolones is not well known. Several in vitro and in vivo study using animals revealed that fluoroquinolones induced oxidative stress by producing reactive oxygen species (ROS).

In vitro studies showed that fluoroquinolones induce an early stimulation of the oxidative metabolism in immature rabbit chondrocytes ${ }^{[13]}$ and differential effect on newly differentiating in vitro human cells. ${ }^{[14,15]}$ Fluoroquinolones are capable of modulating oxidative metabolism of leukocytes and stimulating the production of reactive oxygen species in neutrophils. ${ }^{[16]}$ Perhaps there were no studies that could substantiate the role of free radical in adverse effects caused by fluoroquinolones in patient population and on specified dosage regimen.

The present investigations are focused on studying the oxidative stress induced by Ciprofloxacin, levofloxacin and gatifloxacin in patients with complicated urinary tract infections (UTI), antioxidant status and more importantly the effect of dosage regimen.

\section{MATERIALS AND METHODS}

All chemicals used in study were of analytical grade. CFX, LVX and GTX (Active Pharmaceutical ingredients) were obtained from Dr. Reddy's Laboratories (Hyderabad, India). All solvents were HPLC grade. Acetonitrile, acetic acid, and methanol were purchased from Rankem (Ranbaxy, Mumbai, India. SOD and O-dianisidine were purchased from Sigma St. Louis, USA. Riboflavin was a kind gift from Natco Pharma, AP, India. Thiobarbituric acid was procured from BDH Fine Chemicals, Mumbai, India. CIFRAN $^{\circledR}$ - (Ciprofloxacin $500 \mathrm{mg}$ tablets, Ranbaxy (Pharma), LEVOFAN ${ }^{\circledR}$ (Levofloxacin $500 \mathrm{mg}$ tablets, Sifam Pharma) GATISTAR ${ }^{\circledR}$ (Gatifloxacin — $400 \mathrm{mg}$ tablets, Sifam Pharma) were used in the study.

\section{Instrumentation and chromatography}

Analysis of the plasma samples were carried out using reported method. ${ }^{[17]}$ Chromatography was performed on Prominence (Shimadzu Corporation) HPLC equipment consisting of Jasco FP-920 fluorescence detector, liquid chromatography (LC-10 ADVP), system controller (SCL10AVP), a Photo diode array detection (PDA) system (SPD-M 10AVP). A stainless steel Phenomenex (C-18 $\times 4 \mathrm{~mm}$ column, $250 \mathrm{~mm}$, and $0.25 \mu \mathrm{m}$ ) was used as stationary phase.

Plasma antioxidant status (PAS) was evaluated using specific HPLC method ${ }^{[18]}$ by evaluating the DPPH-free radical scavenging activity in plasma using a LiChrospher ${ }^{\mathbb{R}}$ $100 \mathrm{RP}-18 \mathrm{e}$ column $(250 \times 4 \mathrm{~mm}, 5 \mu \mathrm{M})$. The mobile phase was a mixture of methanol and water $(80: 20, \mathrm{v} / \mathrm{v})$ pumped at a flow rate of $1 \mathrm{~mL} / \mathrm{min}$. The DPPH peaks were monitored at $517 \mathrm{~nm}$.

The estimation of SOD a free radical scavenging enzyme, was performed using the photo oxidation method ${ }^{[19]}$ in the hemolysates. To $2 \mathrm{ml}$ of plasma equal volume of cold deionized water was added. The mixture was centrifuged at $3000 \mathrm{rpm}$ for $15 \mathrm{~min}$, and the SOD activity was measured in the supernatant. To $0.88 \mathrm{ml}$ of riboflavin solution $\left(1.3 \times 10^{-5} \mathrm{mM}\right.$ in $0.01 \mathrm{M}$ potassium phosphate buffer, $\mathrm{pH}$ 
7.5) $60 \mu \mathrm{l}$ of O-dianisidine solution (10 $\mathrm{mM}$ in ethanol) was added. To this $1 \mathrm{ml}$ of distilled water was added and kept away from light. One hundred microliters of the separated SOD was added and optical density (OD) measured at $460 \mathrm{~nm}$ using the spectrophotometer. The cuvette was then transferred to the illumination box (40 Watt white fluorescent tube) for exactly $4 \mathrm{~min}$ and the OD was remeasured against blank containing ethanol in place of enzyme. The SOD was estimated from the standard graph plotted using different concentrations of pure bovine SOD.

Plasma concentrations of malondialdehyde (MDA) as thiobarbituric acid complexes were measured by HPLC. ${ }^{[20]}$ At the beginning of assay, $0.1 \%$ butylated hydroxytoluene a chain breaking antioxidant was added to prevent the peroxidation of lipid. These levels indicate the lipid peroxidation and hence the oxidative stress. Glutathione was estimated in plasma by the reported method in which oxidation of GSH by the sulfhydryl reagent 5,5'-dithio-bis(2-nitrobenzoic acid) (DTNB) to form the yellow derivative 5'-thio-2-nitrobenzoic acid (TNB), measurable at $412 \mathrm{~nm}^{\left[{ }^{[21]}\right.}$

\section{Patient selection}

Patient selection was done on randomized basis from four tertiary hospitals (Sharanya hospitals) in Warangal and Hyderabad, India. All the patients were positive for UTI after the confirmation from culture tests (bacterial count of more than 10,000 CFU per mL of urine, diagnosed as Complicated UTI caused by Escherichia coli, Klebsiella pneumoniae, Enterococcus faecalis, Proteus mirabilis or Psendomonas aeruginosaa). The patients selected after doing physical examination, electrocardiograms, laboratory screening (testing for drugs of abuse, hepatitis and human immunodeficiency virus), serology, hematological and biochemical parameters. All volunteers had normal hepatic, renal functions (mean creatinine clearance of $\left.112.5 \pm 10.4 \mathrm{ml} / \mathrm{min} / 1.73 \mathrm{~m}^{2}\right)$, lipid peroxidation and antioxidant status compared to healthy volunteers. Further inclusion criteria were as follows: No history of gastrointestinal disease or surgery, no medication of any kind within 1 week and no alcohol ingestion within $48 \mathrm{~h}$ of study initiation, no allergy or intolerance to any drugs (especially to quinolones), no blood donation, and no participation in a clinical trial within 60 days of the study. These patients did not receive any antibiotics before initiation of the treatment with fluoroquinolones.

Total of 52 patients were selected with mean age of $45 \pm 11$ years of which 12 were male and 30 were females. The dosage regime was $500 \mathrm{mg}$ of CIFRAN thrice a day, LEVOFAN $500 \mathrm{mg}$ twice a day and GATISTAR $400 \mathrm{mg}$ twice a day (standard dosage regimen for treating complicated UTI infections). All the medications are given orally for 5 days. 16 patients each were on Ciprofloxacin and levofloxacin treatment group and other 20 patients on gatifloxacin. The study was approved by the Institutional Clinical ethics committee (NIPER, Hyderabad, India) and written consents were taken from all the patients participating in the study.

\section{Blood sampling}

Blood sampling was carried out in different phases. Five milliliters blood was collected before initiation of the therapy and on subsequent days $\left(1^{\text {st }}, 2^{\text {nd }}, 3^{\text {rd }}\right.$, $\left.4^{\text {th }}, 5^{\text {th }}\right)$ day of the treatment. Blood was withdrawn at second hour immediately after administering the dose of fluoroquinolones on that particular day. Plasma was separated immediately with addition of anticoagulant and the samples were analyzed for drug concentrations and other biochemical parameters mentioned above.

\section{Data analysis}

All the data are represented as mean values $\pm S D$. Statistical analysis was performed using a two-tailed, unpaired Student's $t$-test. When multiple comparisons were needed, an analysis of variance followed by Student Newman Keuls test using Graph Pad Prism (Version 4) was followed. Values of $P<0.05$ were considered significant.

\section{RESULTS}

The concentrations indicate either the $C_{\max }$ (day 1) or the $\mathrm{C}_{\mathrm{SS}}$ (concentrations at steady state for subsequent doses). Plasma concentrations of CFX, plasma antioxidant status, lipid peroxides, superoxide dismutase (SOD) and glutathione at different time intervals are shown in Table 1. There is no significant difference in the concentration levels either during the loading or maintenance doses. The studies indicate a progressive increase in the lipid peroxide levels at different days. There is significant increase in the lipid peroxide levels from the $1^{\text {st }}$ day $(3.6 \pm 0.34 \mathrm{nM})$ to $5^{\text {th }}$ day $(6.2 \pm 0.94 \mathrm{nM})$, which almost doubled. There is also significant decrease in SOD $(73.3 \%)$ and glutathione (25.5\%) levels with repeated administration of the CFX up to $5^{\text {th }}$ day. Plasma antioxidant status (DPPH) decreased by $77.6 \%$ which is considered extremely significant compared to levels before initiation of therapy.

Similar results were observed in patient group on LVX therapy but moderate decrease in SOD (32.2\%), 
glutathione (15.2\%) and plasma antioxidant status (50.5\%) as mentioned in Table 2. Lipid peroxide levels increased from $3.5 \pm 0.84 \mathrm{nM}$ to $5.1 \pm 0.28 \mathrm{nM}$ which is considered significant. On $5^{\text {th }}$ day, plasma concentration of LVX was found to be $5.78 \pm 0.45 \mu \mathrm{g} / \mathrm{ml}$ compared to $5.24 \pm 0.73$ on day 1. In contrast GTX showed minimal increase in lipid peroxide levels. There was an insignificant alteration observed in SOD, glutathione and plasma antioxidant levels [Table 3].

The studies indicate significant disruption of the antioxidant status that could be because of the depletion of the free radical scavenging enzymes which ultimately resulted in elevated lipid peroxide levels. This sequel is observed with CFX and LVX but not with GTX.

\section{DISCUSSION}

This is the first in human, prospective study to investigate oxidative stress of flouroquinolones in pathological situation and the effect of dosage regimen. The results of this study show that ciprofloxacin and levofloxacin have increased the lipid peroxidation from $3.6 \pm 0.34 \mathrm{nmol} / \mathrm{ml}$ to $6.2 \pm 0.94 \mathrm{nmol} / \mathrm{ml}$ and $3.5 \pm 0.84 \mathrm{nmol} / \mathrm{ml}$ to $5.1 \pm 0.28 \mathrm{nmol} / \mathrm{ml}$ in a time dependent manner. The degree of ciprofloxacin induced lipid peroxidation seen in this study was comparable to that previously reported. ${ }^{[22,23]}$ The effect is greatest 5 days after exposure to ciprofloxacin and levofloxacin therapy, which indicates the formation of reactive oxygen species as in previous studies with fluoroquinolones. ${ }^{[24,25]}$ These results further supported by decrease in plasma antioxidant status by $77.6 \%$ and $50.5 \%$ for ciprofloxacin and levofloxacin respectively.

This increase in oxidative stress may be responsible for the pathological mechanism of tendinitis due to fluoroquinolones. ${ }^{[2]}$ Histological studies have identified ultra-structural abnormalities in tenodocytes and the presence of giant cells, the features being similar to those of overuse injuries. Animal studies have suggested that chelation of magnesium and free radical formation result in oxidative stress, leading to a direct toxic effect on collagen. ${ }^{[27-29]}$ The effect of fluoroquinolones on achilles

Table 1: Plasma concentration of CFX, lipid peroxides, SOD, glutathione and PAS on different days. Values represent mean \pm SD of 16 patients

\begin{tabular}{|c|c|c|c|c|c|}
\hline Day & CFX Con $(\mu \mathrm{g} / \mathrm{ml})$ & LP (nmol/ml) & SOD (I.U) & GLUT $(\mu \mathrm{mol} / \mathrm{L})$ & PAS $^{\circledR}$ (nmol) \\
\hline 0 & & $3.6 \pm 0.34$ & $57.23 \pm 5.2$ & $86 \pm 25$ & $63.4 \pm 5.42$ \\
\hline 1 & $3.34 \pm 0.18$ & $3.8 \pm 0.96$ & $42.56 \pm 4.89^{* *}$ & $402 \pm 28 * * *$ & $51.8 \pm 4.98 * * *$ \\
\hline 2 & $3.42 \pm 0.25$ & $4.2 \pm 0.78$ & $30.25 \pm 5.55^{* * *}$ & $401 \pm 30^{* * *}$ & $32.6 \pm 7.26^{* * *}$ \\
\hline 3 & $3.88 \pm 0.34 * * *$ & $4.8 \pm 1.05^{* * *}$ & $24.23 \pm 5.79 * * *$ & $398 \pm 38^{* * *}$ & $20.9 \pm 6.84 * * *$ \\
\hline 4 & $3.91 \pm 0.19 * * *$ & $5.2 \pm 0.87 * * *$ & $18.34 \pm 6.23 * * *$ & $375 \pm 45^{* * *}$ & $15.4 \pm 4.56^{* * *}$ \\
\hline 5 & $3.88 \pm 0.21^{* * *}$ & $6.2 \pm 0.94 * * *$ & $15.23 \pm 7.01 * * *$ & $364 \pm 41^{* * *}$ & $14.2 \pm 4.21 * * *$ \\
\hline
\end{tabular}

${ }^{\circledR}$ Equivalent to ascorbic acid. ${ }^{*} P<0.05, * * P<0.01,{ }^{* * *} P<0.001$ relative to day 0 for LP, SOD, GLUT, PAS and day 1 for drug concentrations

Table 2: Plasma concentration of LVX, lipid peroxides, SOD, glutathione and PAS on different days. Values represent mean \pm SD of 16 patients

\begin{tabular}{|c|c|c|c|c|c|}
\hline Day & LVX Con $(\mu \mathrm{g} / \mathrm{ml})$ & LP (nmol/ml) & SOD (I.U) & GLUT $(\mu \mathrm{mol} / \mathbf{L})$ & PAS $^{@}($ nmol) \\
\hline 0 & & $3.5 \pm 0.84$ & $59.33 \pm 7.45$ & $473 \pm 29$ & $60.34 \pm 7.89$ \\
\hline 1 & $5.24 \pm 0.73$ & $3.86 \pm 0.46$ & $55.78 \pm 5.23$ & $450 \pm 21 *$ & $58.8 \pm 5.42$ \\
\hline 2 & $5.68 \pm 0.94$ & $3.9 \pm 0.56$ & $50.21 \pm 5.09 * * *$ & $420 \pm 22 * * *$ & $49.82 \pm 8.56^{* * *}$ \\
\hline 3 & $5.71 \pm 0.78$ & $4.2 \pm 0.87$ & $50.23 \pm 4.77 * * *$ & $421 \pm 34 * * *$ & $40.54 \pm 3.27 * * *$ \\
\hline 4 & $5.70 \pm 0.97$ & $4.2 \pm 0.56^{*}$ & $42.75 \pm 4.99 * * *$ & $400 \pm 48 * * *$ & $38.54 \pm 5.85^{* * *}$ \\
\hline 5 & $5.78 \pm 0.45$ & $5.1 \pm 0.28^{*}$ & $40.75 \pm 6.97 * * *$ & $401 \pm 23^{* * *}$ & $30.75 \pm 8.94 * * *$ \\
\hline
\end{tabular}

*P<0.05, ${ }^{* *} P<0.01, * * * P<0.001$ relative to day 0 for LP, SOD, GLUT, PAS and day 1 for drug concentrations

Table 3: Plasma concentration of GTX, lipid peroxides, SOD, glutathione and PAS on different days. Values represent mean \pm SD of 20 patients

\begin{tabular}{|c|c|c|c|c|c|}
\hline Day & GTX Con $(\mu \mathrm{g} / \mathrm{ml})$ & LP (nmol/ml) & SOD (I.U) & GLUT $(\mu \mathrm{mol} / \mathrm{L})$ & $\mathbf{P A S}^{\circledR}(\mathbf{n m o l})$ \\
\hline 0 & & $3.5 \pm 0.84$ & $58.34 \pm 8.21$ & $486 \pm 34$ & $67.44 \pm 3.48$ \\
\hline 1 & $3.61 \pm 0.51$ & $3.66 \pm 0.96$ & $58.43 \pm 5.72$ & $478 \pm 35$ & $62.52 \pm 5.33$ \\
\hline 2 & $3.81 \pm 0.48$ & $3.54 \pm 0.44$ & $57.11 \pm 7.32$ & $471 \pm 46$ & $65.22 \pm 8.75$ \\
\hline 3 & $3.84 \pm 0.39$ & $3.67 \pm 0.72$ & $51.34 \pm 9.88^{*}$ & $460 \pm 41$ & $62.55 \pm 8.64$ \\
\hline 4 & $3.88 \pm 0.69$ & $3.79 \pm 0.48$ & $53.37 \pm 4.89$ & $470 \pm 56$ & $62.89 \pm 5.78$ \\
\hline 5 & $3.84 \pm 0.89$ & $3.74 \pm 0.17$ & $54.69 \pm 5.37$ & $467 \pm 42$ & $62.34 \pm 7.89$ \\
\hline
\end{tabular}

$* P<0.05, * * P<0.01, * * * P<0.001$ relative to day 0 for LP, SOD, GLUT, PAS and day 1 for drug concentrations 
tendon proteoglycans and collagen in rodents showed convincingly that quinolone-induced oxidative stress altered proteoglycan anabolism and oxidized collagen. Pefloxacin treatment for several days induced oxidative damage of collagen type I, with the alterations being identical to those observed in the experimental tendinous ischemia and reperfusion model. ${ }^{[30]}$

The efforts of the endogenous antioxidant enzymes like SOD to remove the continuously generated free radicals initially increase due to an induction but later enzyme depletion occurs by $73.3 \%$ and $32.2 \%$ for ciprofloxacin and levofloxacin respectively, resulting in oxidative cell damage. Hence when the generation of reactive free radicals overwhelms the antioxidant defence, lipid peroxidation of the cell membrane occurs. This causes disturbances in cell integrity leading to cell damage/death. In the present study the repeated administration of CFX (recommended dosage regimen of CFX for UTI) resulted in increase free radical adduct generation by CYP450 mediated metabolism that cumulate and may result in increased $\operatorname{ROS}^{[26]}$ and substantial reduction in antioxidant defence. Perhaps this could be the reason for the adverse effect in particular tendinitis attributed to free radical generation. The present investigations support the pathogenesis of chondrotoxicity explained by the magnesium-chelating properties of these drugs, leading to radical formation and finally to irreversible cartilage lesions. Animal toxicological studies have been published confirming that the quinolone-induced tendopathy is a drug-induced, dose-dependent effect of these agents and profoundly seen with CFX and LVX. ${ }^{[31]}$

In vitro studies on tendon cells also report low toxicity after $24 \mathrm{hr}$ for all fluoroquinolones, but a high-significant tenotoxicity after 48-72 hr. Free radical overproduction was observed for all fluoroquinolones, but significant anion superoxide increased only with pefloxacin and ciprofloxacin. Studies separate two models of fluoroquinolones tenotoxicity: Pefloxacin or ciprofloxacin induced a higher intrinsic tenotoxicity than ofloxacin or levofloxacin. ${ }^{[32]}$ A reported hypothesis indicates that, up on administration of fluoroquinolones the glutathione content falls rapidly so the intracellular antioxidant enzymes lose their ability to modulate the overproduction of $\operatorname{ROS}^{[33]}$ which was also observed in the present study. Gatifloxacin produced lesser oxidative stress and can be consider safe relative to ciprofloxacin and levofloxacin.

\section{CONCLUSIONS}

There was a considerable increase in lipid peroxide levels indicating an enormous oxidative stress. Caution to be taken for patients especially on Ciprofloxacin and Levofloxacin therapy. These studies necessarily warranty the use of exogenous antioxidants as adjuvant in combination with fluoroquinolones, and their benefits should be carefully examined in a controlled clinical setup.

\section{REFERENCES}

1. TSN Database, MRL Pharmaceutical Services1999.

2. Lietman PS. Fluoroquinolone toxicities: An update. Drugs. 1995;49 (Suppl 2):156-63.

3. Hooper DC, Wolfson JS. The fluoroquinolones: Pharmacology, clinical uses, and toxicities in human. Antimicrob Agents Chemother. 1985; 28:716-21.

4. Bethell DB, Hien TT, Phi LT. Effects on growth of single short courses of fluoroquinolones. Arch Dis Child. 1996;74:44-6.

5. Wagai N, Tawara K. Quinolone antibacterial-agent-induced cutaneous phototoxicity: Ear swelling reactions in BALB/c mice. Toxicol Lett 1991;58: 215-23.

6. Sanchez JP, Domagala JM, Hagen SE, Heifetz CL, Hutt MP, Nichols JB, et al. Quinolone antibacterial agents: Synthesis and structure-activity relationships of 8 -substituted quinolone-3-carboxylic acids and 1,8-naphthridine-3-carboxylic acids. J Med Chem. 1988;31:983-91.

7. Fish DN, Chow AT. The clin pharmacokinetics of levofloxacin. Clin Pharmacokinet. 1997;32:101-19.

8. Perry CM., Barman BJ, Lamb HM. Gatifloxacin. Drugs. 1999;58:683-96.

9. Roblin PM, Hammerschlag MR. In-vitro activity of gatifloxacin against Chlamydia trachomatis and Chlamydia pneumoniae. J Antimicrob. Chemother. 1999;44:549-51.

10. Schaumann R, Ackermann G, Pless B, Claros MC, Rodloff AC. In vitro activities of gatifloxacin, two other quinolones, and five nonquinolone antimicrobials against obligately anaerobic bacteria. Antimicrob Agents Chemother 1999;43:2783-6.

11. Christ W, Esch B. Adverse reactions to fluoroquinolones in adults and children. Infect Dis Clin Pract. 1994;3(Suppl 3):168-76.

12. Wolfson JS, Hooper DC. Overview of fluoroquinolone safety. Am J Med 1991;91(Suppl 6A):153-61

13. Thoung GM, Domarle O, Pocidalo J, Hayem G. Effects of fluoroquinolones on cultured articular chondrocytes flow cytometric analysis of free radical production. J Pharmacol Exp Therap. 1996;271:1544-9

14. Montanari MP, Prenna M, Mingoia M, Ripa S, Varaldo PE. In vitro Antibacterial activity of trovafloxacin and five other fluoroquinolones. Chemotherapy 1998;44:85-93.

15. Majtán V, Majtánová L. Postantibiotic effect of some antibiotics on the metabolism of Pseudomonas aeruginosa, J of Basic Microbiol. 1998;38:221-7.

16. Matsumoto T, Takahashi K, Nagafuji T, Kubo S, Sakumoto M, Mochida $\mathrm{O}$, et al. Fleroxacin enhancement of superoxide production by polymorphonuclear leukocytes: The role of protein kinases. Chemotherapy. 1996;42:280-5.

17. Hairui L, Michael BK, Kevin MS. Separation of levofloxacin, ciprofloxacin, gatifloxacin, moxifloxacin, trovafloxacin and cinoxacin by high-performance liquid chromatography: Application to levofloxacin determination in human plasma. J Chromatogr B Analyt Technol Biomed Life Sci. 2002;772:53-63.

18. Chandrasekar D, Madhusudhana K, Ramakrishna S, Prakash VD. Determination of free radical scavenging activity by reversed-phase HPLC: A sensitive screening method for polyherbal formulations. J Pharma Biomed Analysis; 2006;40:460-4

19. Misra HP, Fridovik I. Role of superoxide radicals in the acute auto oxidation of epinephrine; a simple assay for SOD. J Biol Chem. 1972;247:70-5.

20. Yagi KA. Simple fluorometric assay for lipoperoxide in blood plasma. J Biochem Med. 1976;15:212-6.

21. Irfan R, Aruna K, Saibal KB. Assay for quantitative determination of glutathione and glutathione disulfide levels using enzymatic recycling method. Nat Prot 2007;1:3159-65. 
22. Simonin MA, Gegout-Pottie P, Minn A, Gillet P, Netter P, Terlain B. Proteoglycan and collagen biochemical variations during fluoroquinolone-induced chondrotoxicity in mice. Antimicrob Agents Chemother. 1999;43:2915-21.

23. Weyers AI, Ugnia LI, García Ovando H, Gorla NB. Ciprofloxacin increases hepatic and renal lipid hydroperoxides levels in mice. Biocell 2002;26:225-8.

24. Wagai $\mathrm{N}$, Tawara $\mathrm{K}$. Important role of oxygen metabolites in quinolone antibacterial agent-induced cutaneous phototoxicity in mice. Arch Toxicol 1991;65:495-9.

25. Martinez LJ, Sik RH, Chignell CF. Fluoroquinolone antimicrobials: Singlet oxygen, superoxide and phototoxicity. Photochem Photobiol 1998;67:399-403.

26. Van der Linden PD, van Puijenbroek EP, Feenstra J, Veld BA, Sturkenboom MC, Herings RM, et al. Tendon disorders attributed to fluoroquinolones: A study on 42 spontaneous reports in the period 1988 to 1998. Arthritis Rheum 2001;45:235-9.

27. Michiyuki K, Sanae T, Yoko K, Mamoru N. Histological examination on achilles tendon lesions induced by quinolone antibacterial agents in juvenile rats. Toxicol Pathol 1995;23:385- 9.

28. Harrell, RM. Fluoroquinolone-induced tendinopathy: What do we know? South Med J. 1999;92:622-5.

29. Simonin MA, Gegout PP, Minn A, Gillet P, Netter P, Terlain B.
Pefloxacin-induced achilles tendon toxicity in rodents: Biochemical changes in proteoglycan synthesis and oxidative damage to collagen. Antimicrob Agents Chemother. 2000;44:867-72.

30. Gürbay A, Gonthier B, Daveloose D, Favier A, Hincal F. Microsomal metabolism of ciprofloxacin generates free radicals. Free Radic Biol Med 2001;30:1118-21.

31. Casparian JM, Luchi M, Moffat, RE, Hinthorn D. Quinolones and tendon ruptures. South Med J. 2000;93:488-91.

32. Pouzaud F, Bernard BK, Thevenin M, Warnet JM, Hayem G, Rat P. In vitro discrimination of fluoroquinolones toxicity on tendon cells: Involvement of oxidative stress. J Pharmacol Exp Therap 2004;308:394-402.

33. Pouzaud F, Rat P, Pelle B, Pautre MC, Warnet JM. Tendon Cytotoxicity induced by fluoroquinolones (Levofloxacin, Ofloxacin, Pefloxacin, Ciprofloxacin): Investigation of cell death mechanism and oxidative stress on living tenocytes. Antimicrob Agents Chemother. 2000;40:3.

How to cite this article: Talla V, Veerareddy PR. Oxidative stress induced by fluoroquinolones on treatment for complicated urinary tract infections in Indian patients. J Young Pharmacists 2011;3:304-9.

Source of Support: Nil, Conflict of Interest: None declared. 